\title{
THE
}

$11-19-2014$

\section{Two-slot coiled coaxial cable resonator: reaching critical coupling at a reduced number of coils}

\author{
Gerald Hefferman \\ University of Rhode Island, gerry86@uri.edu \\ Zhen Chen \\ University of Rhode Island \\ Tao Wei \\ University of Rhode Island, tao_wei@uri.edu
}

Follow this and additional works at: https://digitalcommons.uri.edu/ele_facpubs

\section{The University of Rhode Island Faculty have made this article openly available.}

Please let us know how Open Access to this research benefits you.

This is a pre-publication author manuscript of the final, published article.

Terms of Use

This article is made available under the terms and conditions applicable towards Open Access Policy Articles, as set forth in our Terms of Use.

\section{Citation/Publisher Attribution}

Hefferman, G., Chen, Z., \& Wei, T. (2014). Two-slot coiled coaxial cable resonator: Reaching critical coupling at a reduced number of coils. Review of Scientific Instruments, 85(11), 115106. doi: 10.1063/ 1.4901593

Available at: http://dx.doi.org/10.1063/1.4901593

This Article is brought to you for free and open access by the Department of Electrical, Computer, and Biomedical Engineering at DigitalCommons@URI. It has been accepted for inclusion in Department of Electrical, Computer, and Biomedical Engineering Faculty Publications by an authorized administrator of DigitalCommons@URI. For more information, please contact digitalcommons-group@uri.edu. 


\title{
Two-slot coiled coaxial cable resonator: reaching critical coupling at a reduced number of coils
}

\author{
Gerald Hefferman, ${ }^{1,2}$ Zhen Chen, ${ }^{1}$ and Tao Wei ${ }^{1, a)}$ \\ ${ }^{1}$ Department of Electrical, Computer, and Biomedical Engineering, University of Rhode Island, Kingston, Rhode Island, 02881, \\ USA \\ ${ }^{2}$ Warren Alpert Medical School of Brown University, Providence, Rhode Island, 02903, USA
}

a) Author to whom correspondence should be addressed. Electronic mail: wei@ele.uri.edu

This paper reports the experimental demonstration of a coiled coaxial cable resonator capable of meeting the critical coupling condition using a reduced number of coils relative to previously reported coiled resonators. By introducing a second slot along the length of the device, a two-slot coiled coaxial cable resonator was fabricated and critical coupling observed at 22 turns. An additional device with one-slot, but otherwise identically constructed, was also fabricated. After 44 turns, the one-slot device had yet to reach critical coupling. An ultrahigh signal-to-noise ratio (greater than $70 \mathrm{~dB}$ ) was observed at critical coupling of the two-slot device. This reduction in number of slots necessary to reach critical coupling, and the corresponding reduction of physical length of the device, makes this demonstration of the control of critical coupling a potentially important step towards the successful application of coiled coaxial cable resonators to microwave communication and robust sensing applications.

\section{INTRODUCTION}

Coaxial cable and optical fiber are two well-understood and ubiquitously utilized methods of guiding electromagnetic waves for telecommunication. These waveguides rely on identical fundamental electromagnetic theory. Engineering differences between the two technologies arise only as a result of the differing frequencies of the electromagnetic waves used by each technology, with infrared and microwave radiation guided by optical fiber and coaxial cable, respectively. As a consequence of these similarities, the recent application of established communication and sensing technologies within the optical regimes to analogous areas in microwave photonics have met with considerable success. ${ }^{1-6}$

The fabrication of coaxial cables with periodic grating structures is a prime example of this trend..$^{1-4}$ By adopting a structure similar to a fiber Bragg grating (FBG), coaxial cable Bragg gratings $(\mathrm{CCBG})$ can be constructed with many of the same physical characteristics as their 
optical counterparts, allowing for distributed strain and temperature sensing along the cable length, while avoiding problems of fragility inherent in optical fiber systems. Due to the fact that microwave wavelengths are orders of magnitude longer than those of infrared light, the size of these CCBG sensors must be significantly longer than a typical FBG ( $\sim \mathrm{m}$ vs. $\mathrm{mm})$, limiting the number of reflection points that can be fabricated in a single CCBG. This constraint results in a substantially reduced signal-to-noise ratio (SNR) for a CCBG as compared to a FBG, limiting the technology's utility as a multiplexed sensor or microwave filter. ${ }^{6}$

A more recent breakthrough has significantly reduced this limitation. Using principles inspired by optical nanowire microcoil resonator $(\mathrm{ONMR})^{7}$ and fiber ring resonator devices, ${ }^{8,9}$ a coiled coaxial cable resonator (CCCR) has been shown to be capable of achieving an SNR hundreds of times greater that of a CCBG. ${ }^{6}$ A CCCR is constructed by coiling a length of coaxial cable in a spring-like geometry around a cylindrical core and milling a channel along one side of the coil, exposing the inner conductor core of the cable. This novel construction allows a fraction of the transmitted microwave energy within the device to couple to neighboring coils and has resulted in SNRs that rival those of an optical FBG. The critical coupling condition can be met by varying the number of coils of a CCCR. Using one slot to allow coupling between coils, it has been shown that critical coupling was achieved at 38 coils for a previously reported CCCR device. ${ }^{6}$

While the overall size reduction from a CCCR over a CCBG is considerable, a reduction in the number of coils necessary to reach critical coupling, and thus overall length, will confer significant additional advantages to current CCCR technology. Chief among these benefits are reduced power loss per device, which allows a larger number of resonators to be multiplexed in series along a single cable, and the increased ease with which individual devices can be 
integrated into microwave communication systems or embedded within larger structures for sensing applications. These are particularly important traits in structural health monitoring, an area where robust strain and temperature sensors would be particularly advantageous. ${ }^{2,3}$ As a result, the development of CCCR devices with reduced geometries, while continuing to meet critical coupling conditions, is an important engineering goal.

This manuscript describes a method of constructing a CCCR with this reduced geometry. By adding a second channel along the length of a CCCR, critical coupling is reached at a reduced number of cable turns relative to a similarly constructed one-slot CCCR, attributed to the fact that, equivalently, the coupling coefficient for a single turn in the two-slot CCCR increases. While changing the slot width of a one-slot CCCR device does result in a change in coupling coefficient, this change is periodic. ${ }^{10}$ Consequently, the addition of a second slot was investigated as a promising approach to increasing the effective coupling coefficient of a CCCR device. Experimental data from both a two-slot and a one-slot CCCR were collected and the results are presented below. Additionally, simulation data using a previously reported electromagnetic model for a CCCR device are in close agreement with experimentally observed device behavior.

\section{EXPERIMENTAL RESULTS}

In order to experimentally quantify the differing characteristics of a two-slot versus a one-slot CCCR, both types of devices were constructed and investigated. Figure 1 illustrates a general two-slot CCCR device schematic. The two-slot CCCR was constructed by wrapping a length of coaxial cable (RG-58) around an aluminum core $12.6 \mathrm{~mm}$ in diameter, creating a spring-like geometry 45 coils in length. A computer-controlled milling machine (Sherline Model 5400) 
fitted with a 3/16 inch end mill was used to cut two slots along the length of the device. The two slots were positioned $180^{\circ}$ apart across the coil diameter of the CCCR. This geometry allows the lengths between slots to be made approximately identical, avoiding the addition of a further phase-matching condition to the system. Differing relative slot positions will be systematically investigated in subsequent studies. Both slots were milled to approximately half of the crosssectional diameter of the coaxial cable, removing a portion of exterior insulator, outer conductor, interior dielectric layer, and exposing the inner conductor core. The two slots were milled incrementally; as the slot on side A was extended by a single coil length, the device was rotated and the slot on side B extended by the same distance. In this way, both slots advanced in step during fabrication and data collection, with the final slot lengths extending across 44 coils.

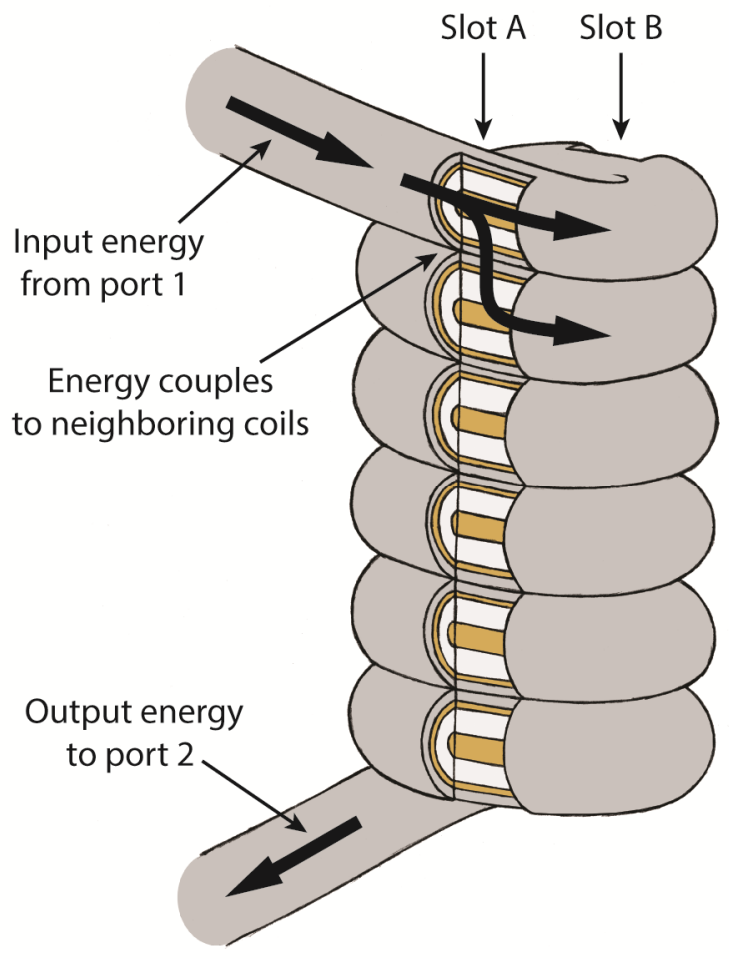

FIG 1. Schematic of the two-slot CCCR device 
A one-slot CCCR was constructed in a similar manner, using the same type and length of coaxial cable, metal core, number of coils, and slot depth and width. As with the two-slot CCCR, the slot along the one-slot CCCR device was milled to a length of 44 coils in total.

Transmission spectral data were collected during the fabrication of both CCCR devices using a vector network analyzer (Agilent N3383A). At each coil-length slot extension, data were recorded across a span of $300 \mathrm{kHz}$ to $6.0 \mathrm{GHz}$ at 3201 linearly-spaced points with a $1.00 \mathrm{kHz}$ intermediate frequency bandwidth (IFBW).

(a) One-slot CCCR

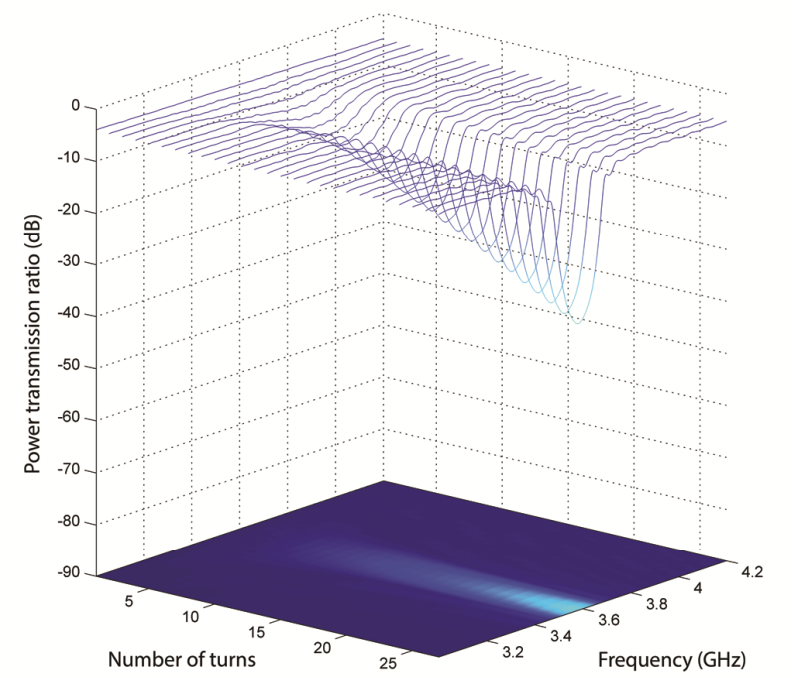

(b) Two-slot CCCR

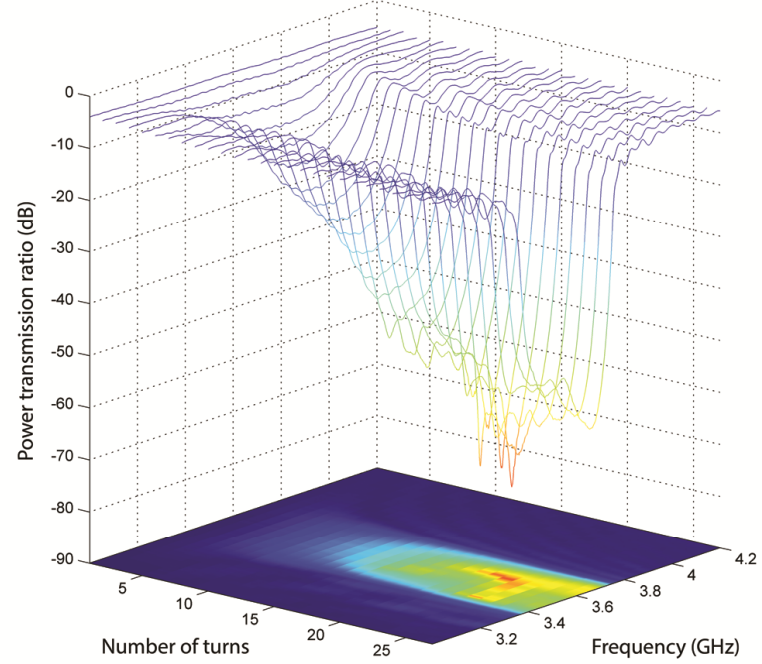

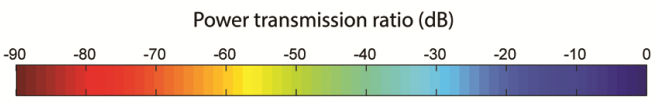

FIG 2. Transmission spectra of both one-slot and two-slot CCCR devices as a function of slot length (number of turns)

Figure 2 illustrates the transmission spectra recorded from each of the two CCCR devices as a function of slot length (number of turns) from $3.0 \mathrm{GHz}$ to $4.2 \mathrm{GHz}$. Both CCCR devices exhibit a strong peak at approximately $3.6 \mathrm{GHz}$, which corresponds to the total linear distance of one 
circulation around a single coil $(5.6 \mathrm{~cm})$. Critical coupling at this frequency was reached using the two-slot CCCR after 22 coils. Here, the peak at $3.606 \mathrm{GHz}$ reached $-72.01 \mathrm{~dB}$ relative to input power. At the same number of coils, the one-slot CCCR exhibited a peak at approximately the same frequency $(3.583 \mathrm{GHz})$ but at only $-27.76 \mathrm{~dB}$ relative to input power. Importantly, the peak level continued to drop for the one-slot CCCR as more coils were included, indicating continued undercoupling. In the case of the two-slot device, undercoupling was seen from 1 to 21 slots, critical coupling at 22 slots, and overcoupling at $\geq 23$ slots. Additionally, ripples are evident in the spectra of the two-slot CCCR device. These ripples are speculated to be the result of cable manufacturing artifact; by using higher-quality cable and increased manufacturing precision, these artifacts are anticipated to be reduced in further experimental tests. These results demonstrate that the introduction of a second slot during fabrication of a CCCR reduces the number of turns necessary to reach critical coupling.

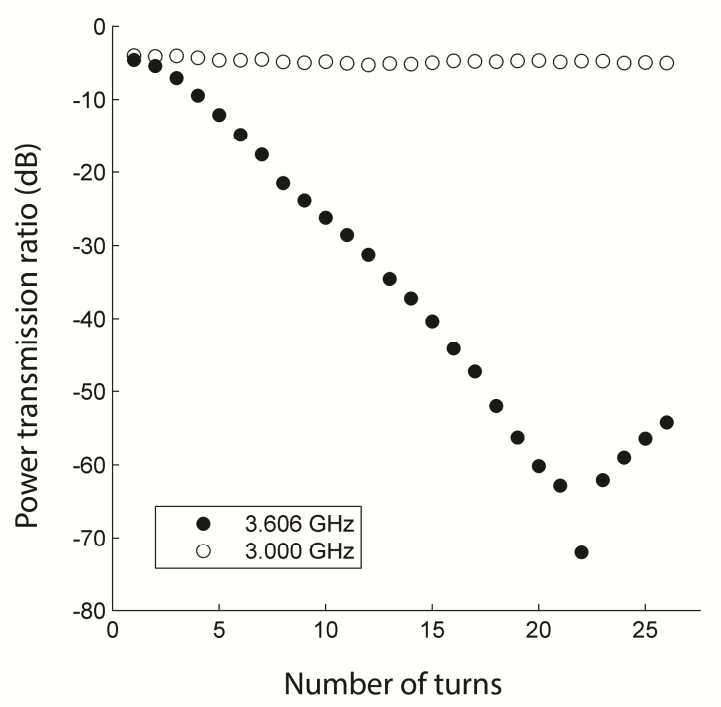

FIG 3. Power transmission ratios as a function of number of turns at both resonant frequency $(3.606 \mathrm{GHz})$ and non-resonant frequency $(3 \mathrm{GHz})$ of the two-slot CCCR 
Importantly, power loss through the two-slot CCCR at non-resonance frequencies was only minimally increased as the slot number was increased. Figure 3 illustrates these results; as slot length increases, the loss at $3.000 \mathrm{GHz}$, a non-resonance frequency, increases by less than $1.4 \mathrm{~dB}$ over 26 coils (52 slot openings). Thus, cable transmission loss is the main contributor to loss through the CCCR, which is proportional to the length of the cable.

\section{THEORECTICAL MODELING AND SIMULATION RESULTS}

In order to theoretically justify the addition of a second slot as a method of reducing the overall length of a CCCR, a previously reported model by Huang et al. was adapted to simulate both one-slot and two-slot coiled resonator devices. ${ }^{6}$ This technique models the CCCR as an effective $2 \times 2$ coupler and ring resonator in combination. The principal departure from the originally reported model that allows it to accommodate a two-slot device concerns the effective transmission coefficient. For a one slot model,

$$
t_{\text {eff }}=t^{n}
$$

where $t_{\text {eff }}$ is the effective transmission coefficient for a one-slot CCCR of $n$ turns and $t$ and is the transmission coefficient for a single coil turn. A two-slot CCCR contains two slots per coil turn. As a result, the equivalent coupling coefficient for one coil turn increases accordingly. Importantly, because the majority of microwave energy within the slot sections of the device remains within the dielectric material, the propagation velocity through both slot and connecting regions of the CCCR can be approximated as being identical, eliminating the need to model a propagation delay between the two slots. This approximation is further justified by the considerably smaller slot width relative to pitch length of the CCCR device. Therefore, in the case of a two-slot device, the effective transmission coefficient is defined as

$$
t_{e f f}=t^{2 n}
$$


Using this modelling technique, simulation data for one-slot and two-slot CCCR devices were calculated. In the simulation, the transmission coefficient $t$ was set to 0.995 at around $3 \mathrm{GHz}$, which was calculated by a commercial finite element EM software; the pitch, or linear distance of a single coil turn, was set to $5.6 \mathrm{~cm}$; the loss per circulation was set as 0.9777 ; and the propagation velocity of the microwave energy in the coaxial cable was set to $2.0 \times 10^{8} \mathrm{~m} / \mathrm{s}$. Modeling results were calculated for 3201 linearly-spaced frequencies from $300 \mathrm{kHz}$ to $6 \mathrm{GHz}$ and from 1 to 50 coils.

(a) One-slot CCCR

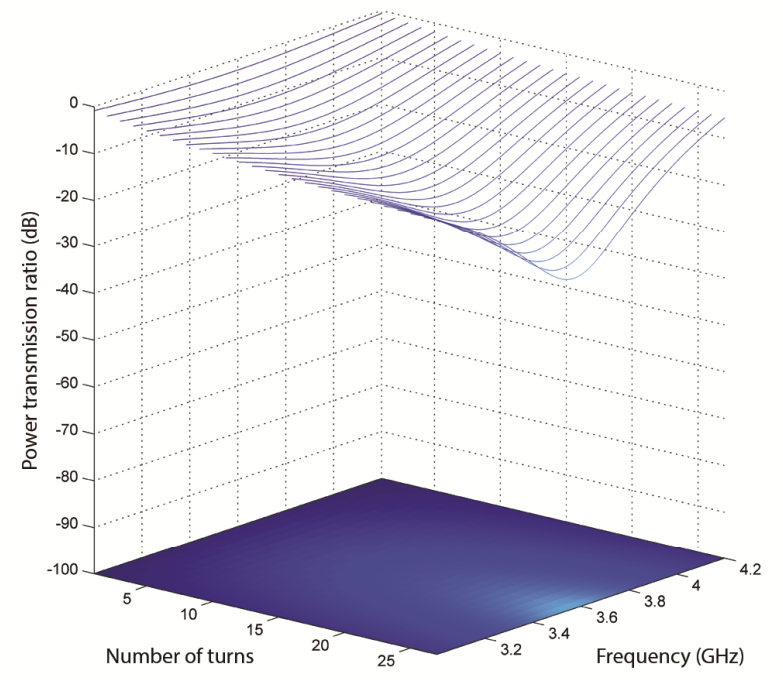

(b) Two-slot CCCR

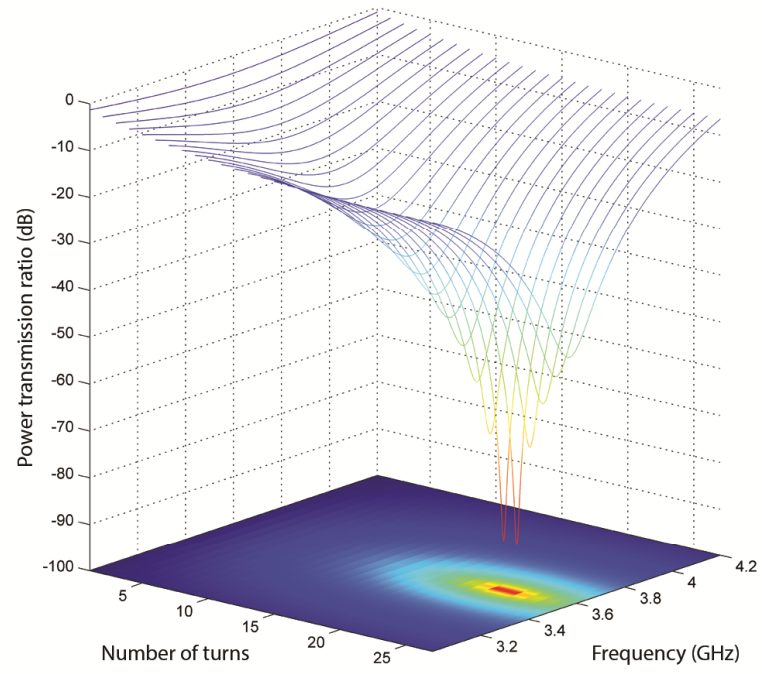

Power transmission ratio $(\mathrm{dB})$

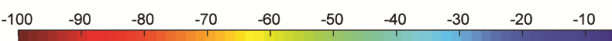

FIG 4. Simulated transmission spectra for both one-slot and two-slot CCCR devices as a function of number of coil turns

Figure 4 illustrates the simulated transmission spectra for the two CCCR devices as a function of number of coil turns. As expected, the two-slot CCCR reached critical coupling at a reduced number of slots (23 turns) as compared to the one-slot device (44 turns). The trend evidenced by the simulation results, that the critical coupling condition is met at fewer turns for a two-slot 
CCCR relative to a one-slot device when all other characteristics are held constant, is in good agreement with experimental observation.

\section{CONCLUSIONS AND DISCUSSION}

The experimental results and simulation data above indicate that the use of two slots in the fabrication of a CCCR reduces the number of coils necessary for the device to meet critical coupling condition relative to a single-slot CCCR, while maintaining ultrahigh SNR. This reduction in the number of coils, and the corresponding reduction in the overall length of the device, confers a number of considerable benefits to CCCR devices that make them increasingly attractive targets for further development and eventual application to communication and sensing applications in the microwave domain.

Chief among these potential areas is the use of a CCCR device as an embedded, multiplexed strain and temperature sensor for structural health monitoring. Due to the substantially increased elasticity, and thus survivability, of coaxial cable as compared to optical fiber, CCCR devices hold particular promise as a method of monitoring elements of critical infrastructure. The practical benefit of this increased robustness is made particularly clear during times of natural disaster, when the need for accurate, real-time structural health information may be most acute. In this context, a reduction in the size of CCCR sensors confers two key advantages: 1) since loss along a coaxial cable is directly proportional to cable length, as evidenced by Figure 3, a reduction in the size of each CCCR allows for more devices to be multiplexed along the same cable while requiring no additional power to maintain a robust signal to noise ratio, and 2) reducing the dimensions of a CCCR allows it to be more easily embedded within a structural element without compromising that element's structural integrity, a key benefit allowing CCCR 
devices to be embedded within a greater variety of buildings and infrastructure. In this way, a reduction in the number of coils necessary to reach critical coupling within a CCCR has direct practical benefits to an area of sensing technology with considerable economic and societal benefit.

\section{ACKNOWLEDGMENTS}

This research was sponsored in part by NSF CCF-1439011.

\section{REFERENCES}

${ }^{1}$ T. Wei, S. Wu, J. Huang, H. Xiao and J. Fan Applied Physics Letters 99, (2011).

${ }^{2}$ J. Huang, T. Wei, X. Lan, J. Fan and H. Xiao, Proceedings of SPIE 8345, Sensors and Smart Structures Technologies for Civil, Mechanical, and Aerospace Systems 2012, (Society of PhotoOptical Instrumentation Engineers).

${ }^{3}$ J. Huang, T. Wang, L. Hua, J. Fan, H. Xiao and M. Luo Sensors 13, (2013).

${ }^{4}$ P. Shi, R. Gao, S. Liu and J. Zhao Sensors and Actuators A: Physical 203, (2013).

${ }^{5}$ J. Huang, T. Wei, J. Fan and H. Xiao Review of Scientific Instruments 85, (2014).

${ }^{6}$ J. Huang, T. Wei, T. Wang, J. Fan and H. Xiao Review of Scientific Instruments 85, (2014).

${ }^{7}$ M. Sumetsky Opt. Express 12, (2004).

${ }^{8}$ A. Yariv Photonics Technology Letters, IEEE 14, (2002).

${ }^{9}$ J. E. Heebner and R. W. Boyd Opt. Lett. 24, (1999).

${ }^{10}$ D. M. Pozar, Microwave engineering (J. Wiley, Hoboken, NJ, 2005) p.354. 